\title{
Effect of resin/hardener stoichiometry on electrical behavior of epoxy networks
}

\author{
F. N. Alhabill, R. Ayoob, T. Andritsch and A. S. Vaughan \\ Tony Davies High Voltage Laboratory \\ University of Southampton, Southampton, UK \\ E-mail: fna1g13@soton.ac.uk
}

\begin{abstract}
By changing the ratio of resin to hardener, a series of epoxy resin samples has been produced with differing network structures and different retained chemical functionalities. The resulting materials were characterized by thermal analysis, dielectric spectroscopy, DC conductivity, and DC and AC breakdown strength measurements, to explore the effect of network structure and chemical composition on molecular dynamics and electrical properties. Differential scanning calorimetry showed that the glass transition temperature is primarily determined by the crosslinking density and indicates that, under the range of conditions employed here, side reactions, such as etherification or homopolarization, are negligible. Conversely, changes in DC conductivity with resin stoichiometry appear to occur as a result of changes in the chemical content of the system, rather than variations in network structure or dynamics. Specifically, we suggest that the DC conductivity is markedly affected by the residual amine group concentration in the system. While DC conductivity and DC breakdown appear broadly to be correlated, AC breakdown results indicated that this parameter does not vary with changing stoichiometry, which suggests that the AC and DC breakdown strengths are controlled by different mechanisms.
\end{abstract}

Index Terms - Epoxy, stoichiometry, glass transition, FTIR, dielectric response, DC conductivity, DC breakdown strength, AC breakdown strength.

\section{INTRODUCTION}

EPOXY resins (ERs) are thermosetting polymers, which are commonly used as adhesives or coatings in a wide range of different industries, e.g. in aerospace and automotive industries $[1,2]$. They find their use as an insulating dielectric in different applications as well, but mainly as the main polymeric insulation in large power generators, switchgear, cast resin transformers, cable terminations and bushings. These applications can have quite diverse working conditions; ERs are popular due to their high resistance to chemicals and their broad range of operating temperatures. The electrical properties of an insulating material are significantly affected by environmental variables such as moisture and operating temperature. Whether the application is AC or DC also makes a difference. Accordingly, each application would require different electrical properties to run optimally, instead of using the one-size-fits-all approach. For example, for applications involving a high level of moisture exposure, reducing the moisture uptake or its effect on the dielectric properties is essential. Better understanding of the mechanisms which control the electrical properties of epoxy networks can help in tailoring and optimizing such materials to fit a particular application. Furthermore, such understanding is fundamental for improving the dielectric performance of these materials.

Although many studies have investigated the electrical and mechanical properties of ER, these studies usually focus on ER cured with a stoichiometric formulation. Nevertheless, a number of reported studies have considered the effect of network structure and resin/hardener stoichiometry on mechanical properties [3-9]. However, very few studies of the effects of stoichiometry on dielectric properties have been reported [10]. Changing the resin/hardener ratio results in changes in the crosslinking density and, consequently, the network structure of the cured epoxy. In addition, this will also affect the functional groups that are retained within the network: for example, in amine-cured systems, using formulations with excess hardener will increase the residual amine group content, whereas using formulations with excess resin will likely lead to an increase in epoxy group content. Furthermore, changing the crosslinking density will affect the concentration of hydroxyl groups that result from the reaction between epoxy and amine groups. Therefore, analyzing the effect of resin/hardener stoichiometry on the electrical properties can give insights on how the network structure and the chemical composition each affect the electrical behavior of the bulk material.

In the last two decades a burgeoning interest has been shown in enhancing the dielectric performance of epoxy networks by filling them with a range of nanofillers, such as silica $[10,11]$, boron nitride $[12,13]$ and silicon nitride [14]. Nanofillers are characterized by their high surface area and are often covered 
with different functional groups, e.g. hydroxyl and amine groups. Once the nanofiller is introduced into the polymer matrix, these groups may interact with the active functionalities existing in the resin and/or the hardener and, consequently, can change the effective resin/hardener stoichiometry. This would explain the different dielectric properties that different researchers find for what, at first glance, appear equivalent polymer-filler-combinations $[15,16]$. Therefore, investigating the impact of changing the resin/hardener stoichiometry on the unfilled epoxy would be beneficial for identifying any stoichiometric effect which might be imparted by the addition of the nanofiller and could help in determining the underlying mechanisms that control the behavior of nano-filled epoxy networks.

This study, as a precursor to considering the impact of nanofiller surface chemistry on epoxy curing, and consequent material properties, set out to investigate the effect of resin/hardener stoichiometry and to consider the influence of the chemical content (represented by amine, epoxy and hydroxyl groups) and the network structure on the electrical behavior of the resulting material. The potential benefits of using non-optimal stoichiometries on dielectric properties are also explored. The effect of stoichiometry on the moisture uptake and the effect of the later on the electrical properties of epoxy networks will be the subject of future work.

\section{EXPERIMENTAL}

\subsection{MATERIALS AND SAMPLES PREPARATION}

The epoxy resin DER-332, supplied by Sigma Aldrich, was used for all samples. This resin is based on dyiglycidyl ether of bisphenol-A (DGEBA), with an epoxy equivalent molar mass of $\sim 174 \mathrm{~g} / \mathrm{mol}$; its chemical structure is illustrated in Figure 1a. The hardener used to cure this resin was Jeffamine D-230, supplied by Huntsman. This has an amine hydrogen equivalent molar mass of $60 \mathrm{~g} / \mathrm{mol}$ and its chemical structure is shown in Figure $1 b$. From this, it is evident that each terminal amine group can react with two epoxy groups, to give the network node structure depicted in Figure 1c. In addition, each epoxy/amine hydrogen reaction also results in the production of a hydroxyl group, which may subsequently react with another epoxy group to generate an ether linkage. The rate of this reaction depends on two factors: the temperature during the curing process and the relative concentration of hydroxyl and amine groups in the system $[17,18]$.

Based on the epoxy and amine equivalent molar masses provided by the manufacturers, the resin : hardener mass ratio should be $1000: 344$. Ideally, this ratio should result in a fully crosslinked network, where no epoxy or amine groups are left unreacted. This theoretical ratio will be taken as a reference and termed the stoichiometric or optimum ratio. At this point, it is convenient to define a new parameter, the hardener percentage $(H P)$, as seen in (1),

$H P=\frac{\text { mass of the hardener used }}{\text { hardener stoichiometric mass }} \%$

where the hardener stoichiometric mass equals the resin mass multiplied by the stoichiometric ratio (344/1000). Thus, formulations with $H P>100 \%$ contain excess amine groups and are termed amine rich samples. Analogously, formulations with $H P<100 \%$ are called epoxy rich samples. Throughout the paper, the samples are identified by their HP. To give some physical meaning to these numbers, consider the case where $H P=120 \%$, whereupon the resulting network theoretically contains $\sim 5 \times 10^{23}$ residual amine groups per meter cube; at $H P=80 \%$ the epoxy system should contain $\sim 6 \times 10^{23}$ residual epoxy groups per meter cube. Thus, in both cases, the average separation between these residual moieties would be $\sim 10 \mathrm{~nm}$.

Sample preparation starts by mixing the required masses of the resin and the hardener with a magnetic stirrer for $15 \mathrm{~min}$, after which time, the mixture is degassed for $20 \mathrm{~min}$, before being injected into a stainless steel mold. Based on the manufacturer's instructions, all samples were cured at $80^{\circ} \mathrm{C}$ for $2 \mathrm{~h}$ and then post-cured at $125^{\circ} \mathrm{C}$ for $3 \mathrm{~h}$. Each sample type was produced in two thicknesses: $70 \pm 5 \mu \mathrm{m}$ for dielectric breakdown measurements and infrared spectroscopy, and $200 \pm 10 \mu \mathrm{m}$ for dielectric spectroscopy and DC conductivity measurements. All samples were stored under dry conditions in a vacuum desiccator for at least two weeks before any measurement, to remove any absorbed water.

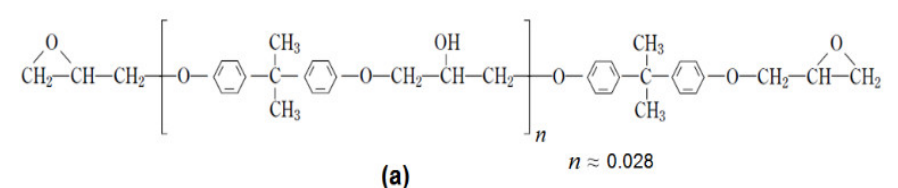

(a)

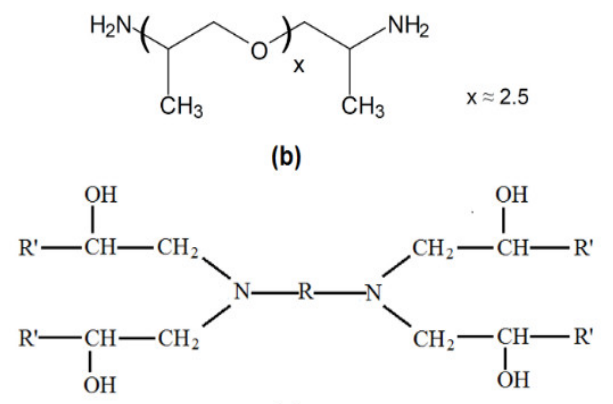

(c)

Figure 1. Chemical structure of (a) DER 332, (b) Jeffamine D-230; (c) a simple representation of a Jeffamine D-230 molecule crosslinked with four epoxy groups.

\subsection{GLASS TRANSITION AND NETWORK STRUCTURE}

The glass transition was observed using a Perkin Elmer DSC 7 differential scanning calorimeter (DSC). Two DSC scans were performed on each sample, around $10 \mathrm{mg}$ in mass, using a heating rate of $10{ }^{\circ} \mathrm{C} / \mathrm{min}$. The measurements were repeated three times for each material, in order to determine the uncertainties in these measurements. The glass transition process was characterized using four parameters: the glass transition temperature $\left(T_{g}\right)$, the glass transition width $\left(\Delta T_{g}\right)$, the heat capacity increase $\left(\Delta C_{p}\right)$ and the enthalpy relaxation area. The enthalpy relaxation area equates to the area of the endothermic peak superimposed upon the glass transition in the first DSC scan, which corresponds to the energy input associated with driving the network segments towards 
structural equilibrium [19]. The enthalpy relaxation area was calculated by subtracting the second DSC scan from the first, as illustrated in Figure 2 [19]. The other three parameters were extracted from the second DSC scan, in the absence of enthalpy relaxation effects.

The extent of epoxy reaction was observed using a Perkin Elmer Spectrum GX Fourier transform infrared (FTIR) spectrometer. The FTIR spectrum of each material was collected from $580 \mathrm{~cm}^{-1}$ to $7800 \mathrm{~cm}^{-1}$ in transmission mode; 32 scans were performed at $4 \mathrm{~cm}^{-1}$ resolution.

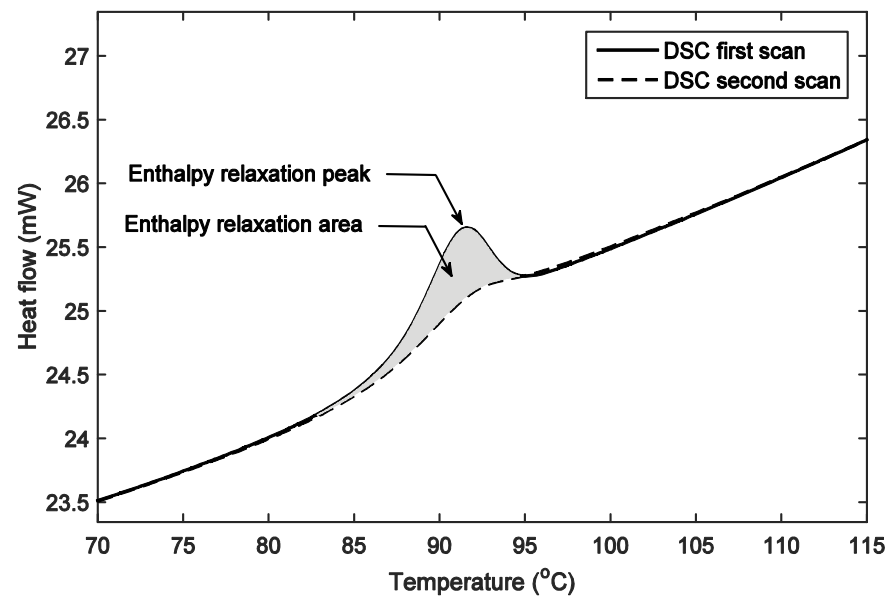

Figure 2. Typical DSC first and second scans for $H P=100 \%$, showing the enthalpy relaxation peak.

\subsection{ELECTRICAL PROPERTIES}

A Solatron 1296 dielectric interface along with a Schlumberger SI 1260 impedance/phase gain analyzer was used to measure the complex permittivity, following procedures outlined in IEC 60250:1969. Initially, a thin layer of gold was sputter coated onto both sides of each specimen, in order to improve the contact between the sample and the electrodes of the measurement cell. The measurement cell consists of two circular parallel electrodes with a diameter of $30 \mathrm{~mm}$ in addition to guard electrodes to reduce fringing effects and to maintain a uniform electric field between the electrodes. The voltage applied to the electrodes was a sinusoidal wave of $7 \mathrm{~V}$, and the temperature of the measurement cell was room temperature $\left(\sim 23^{\circ} \mathrm{C}\right)$.

The DC conductivity of each system was established by measuring the current passing through a specimen placed between two opposing circular electrodes, $30 \mathrm{~mm}$ in diameter. For each sample, the measurement continued for $2 \mathrm{~h}$ at a sampling rate of one measurement per minute. Although such a procedure normally results in a monotonic decrease in the measured current, in this study, the current was found to decrease somewhat over the first $5 \mathrm{~min}$, but remain constant within the noise limits thereafter. In view of the form of these results, inclusion of the raw time dependent data is not warranted; rather, the quoted DC conductivity value for each sample corresponds to the average of all measurements. As above, all samples were initially gold sputtered on both sides, to improve sample/electrode contact.

DC and AC breakdown measurements were performed in accordance with ASTM D3755-14 and ASTM D149 respectively. The sample was placed between two steel ball bearing electrodes, $6.3 \mathrm{~mm}$ in diameter, which were changed every 5 measurements to avoid pitting influencing the derived data. The test cell was immersed in silicone oil (Dow Corning 200/20CS) to prevent flashover. The voltage ramp rate was 50 $\mathrm{V} / \mathrm{s}$ for AC breakdown measurements and $100 \mathrm{~V} / \mathrm{s}$ for DC breakdown measurements, and the testing occurred at room temperature. While such protocols allow many breakdown measurements to be performed in a short period of time, it is important to note that the resulting data can only be used for comparative purposes; the values obtained cannot be interpreted in any absolute sense. Two-parameter Weibull distributions were generated for analysis of the resulting data.

\section{RESULTS AND DISCUSSION}

\subsection{GLASS TRANSITION AND NETWORK STRUCTURE}

No exothermic peak relating to additional curing reactions was observed in any of the DSC measurements, which indicates that the employed curing schedule leads to a maximum possible curing degree. Figure 3 shows the glass transition temperature as a function of hardener percentage and, as would be expected, the theoretical stoichiometric ratio $(H P=100 \%)$ leads to the highest $T_{g}$. Deviating from the stoichiometric ratio, $T_{g}$ decreases but not in a symmetric way: $T_{g}$ decreases more sharply for the epoxy rich samples. Comparable variations in $T_{g}$ have been reported previously $[4,8]$ for similar resin and hardener systems.

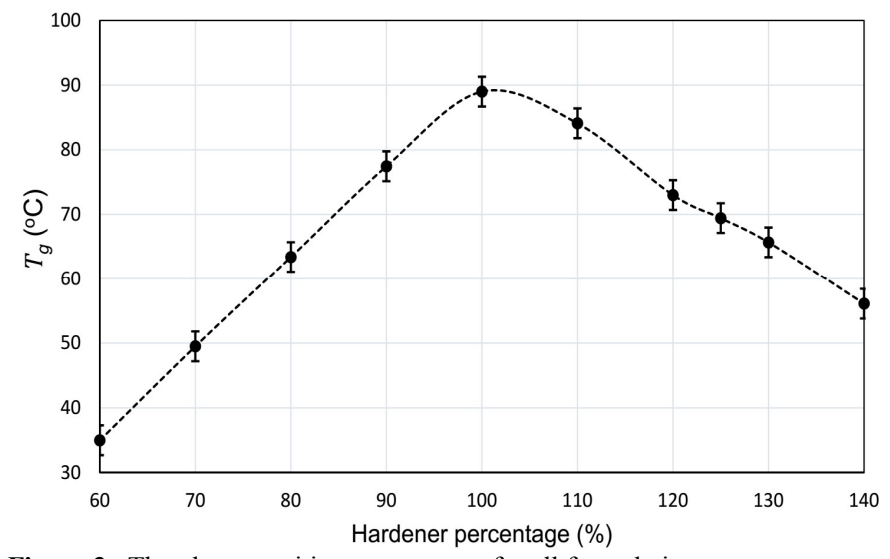

Figure 3. The glass transition temperature for all formulations.

For crosslinked polymers, $T_{g}$ can be affected by the stiffness of the segments between crosslinking points, the free volume content and the crosslinking density. Considering first the rigidity of the segments between the crosslinking joints, the polyether diamine molecules in the Jeffamine D-230 are more flexible than the DGEPA molecules in the DER-332, which would suggest that samples containing an excess of epoxy molecules should exhibit a higher $T_{g}$. However, the results presented above demonstrate the reverse, which implies that the segment rigidity is not the dominant factor.

The free volume content depends on the regularity of the polymer chains and their ability to pack efficiently [5]. Both the resin and the hardener used in this study are linear aliphatic 
molecules, which does not enable easy correlation between the regularity of the polymer chains and the free volume content of the system. However, previous work has concluded that crosslinking imposes steric restrictions on the polymer segments near the crosslink junctions, which reduces the packing efficiency and consequently increases the local free volume content $[5,20]$. Indeed, for a similar resin and hardener system to the one used here, Morgan et al [4] reported that the cured epoxy corresponding to the stoichiometric formulation had a minimum in the macroscopic density, compared with other formulations. This would suggest that the system with $H P=100 \%$ contains more free volume. However, at temperatures near $T_{g}$, crosslinking can limit the divergence of chain segments, which consequently reduces the thermally induced free volume within the system [20]. As a consequence, the influence of free volume content on $T_{g}$ is dominated by the crosslinking density and, therefore, we suggest it is this factor that largely determines the form of the data shown in Figure 3. This assertion is supported by dynamic mechanical characterization data reported elsewhere [4] for the same resin, albeit cured with a similar, but different, amine hardener (Jeffamine T-403). This study showed that the crosslinking density and $T_{g}$ are well correlated and follow similar trends; both decrease more sharply for formulations containing excess epoxy. The asymmetry in the derived crosslinking density might be related to the fact that each molecule in the diamine hardener has four active hydrogen atoms, whereas each molecule in the DEGBA resin has two active epoxy groups.

FTIR spectra obtained from specimens prepared from different stoichiometric ratios are presented in Figure 4. For samples where $H P<100 \%$, an absorption peak is evident near $915 \mathrm{~cm}^{-1}$, which is assigned to the resin's epoxy groups [21]; the intensity of this increases monotonically with decreasing $H P$ in this composition range. For samples with the stoichiometric formulation or with an excess of hardener ( $H P>100 \%)$, this peak disappears. Thus, we conclude that for epoxy rich samples, a fraction of the epoxy groups is left unreacted while, in the amine-rich systems, all are consumed during curing. Other key FTIR features are located in the wavenumber range 3150 to $3650 \mathrm{~cm}^{-1}$. For epoxy rich samples, this feature is related to the hydroxyl groups discussed in connection with Figure 1c, which result in a broad absorbance peak centered at $\sim 3420 \mathrm{~cm}^{-1}$ [22]. For amine rich samples, additional overlapping absorption centered at $\sim 3300 \mathrm{~cm}^{-1}$ occurs, which is associated with residual amine groups; the appearance of this as a single peak at a relatively low wave number is indicative the presence of secondary (rather than primary) amine groups [22]. This peak is evident only in amine rich formulations, where its intensity increases monotonically with increasing $H P$ above $100 \%$, thereby indicating the presence of retained $\mathrm{N}-\mathrm{H}$ groups (i.e. unreacted secondary amines) in these formulations. Compared with the stoichiometric formulation, the FTIR spectra show that the breadth and strength of the hydroxyl peak decrease for all other formulations, with a more pronounced decrease for formulations with $H P<100 \%$. Hence, the FTIR spectra indicate that the concentration of the hydroxyl group decreases for all non-stoichiometric formulations, particularly for epoxy rich formulations. The hydroxyl concentration will be revisited next section. The increase in absorption in the range

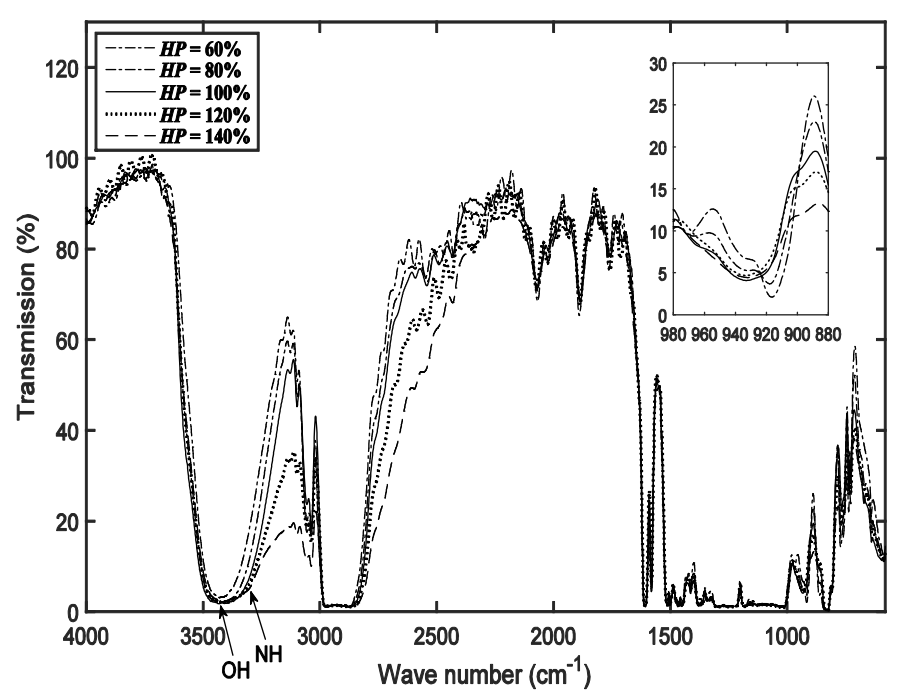

Figure 4. FTIR spectra for selected samples.
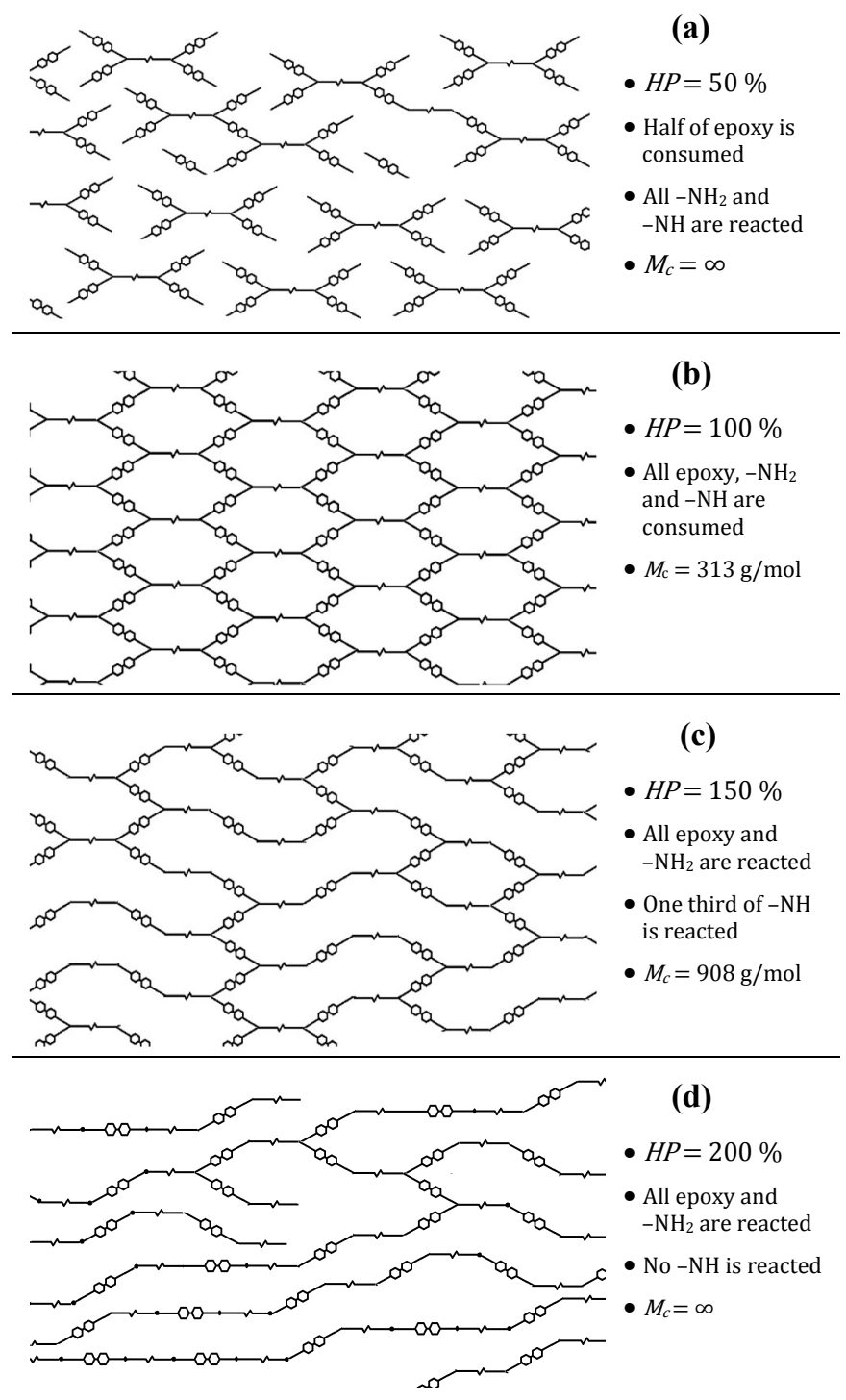

$\checkmark \times$ : DEGBA molecule (DER-332), て : Diamine molecule (Jeffamine D-230)

Figure 5. A schematic representation of the network structure as a function of hardener percentage. 
$2500-2800 \mathrm{~cm}^{-1}$ as a function of $H P$ is attributed to an increase of the aliphatic $\mathrm{C}-\mathrm{H}$ bonds present in the hardener, even though absorption around $2900 \mathrm{~cm}^{-1}$ is saturated [23, 24].

On the basis of the spectral data presented above, it is possible to represent, schematically, the effect of resin stoichiometry on network structure. Figure 5 does this for a number of $H P$ values in the range $50 \%$ to $200 \%$ and is based on the DER-332 resin behaving as a bifunction monomer, being terminated with an epoxide group at either end, while the hardener is a quarto-functional moiety. It is assumed that the reaction rate of the primary amine is higher than that of the secondary amine, as evinced by the FTIR data shown above and also reported by Morgan et al [4], and that no epoxy group is left unreacted while there is an available amine group and vice versa (i.e. complete reaction). For formulations containing excess epoxy $(H P<100 \%)$, all the primary and secondary amines will react, leaving a fraction of unreacted epoxy groups within the network, as terminal end groups or unreacted resin (see Figure 5a). In the case of formulations that contain an excess of hardener, the primary amine hydrogens will react first and, thus, in the case of $H P=150 \%$ (see Figure $5 \mathrm{c}$ ), all primary amine hydrogen will react, while only one third of the secondary amine hydrogen will be consumed. Based on the above assumptions, the average molecular mass between crosslinks $\left(M_{c}\right)$ is calculated and shown in the same figure.

Many studies have indicated that, in addition to epoxy/amine reaction, additional chemical processes can also occur during the curing process. For example, the hydroxyl groups generated by the epoxy/amine reaction may react with epoxy groups in the resin to form ether bonds, or the epoxy groups may react with each other (homopolymerization), particularly at high temperature [27, 28]. However, the FTIR data presented above provide no evidence for this. Also, the dependence of $T_{g}$ on amine/epoxy stoichiometry discussed above does not imply that the extent of such reactions is significant since, particularly for epoxy rich systems, the existence of such reactions would lead to additional crosslinking processes that would tend to hinder the acute decrease in $T_{g}$ observed with decreasing $H P$. Nevertheless, to investigate this hypothesis further, selected samples were postcured at $160{ }^{\circ} \mathrm{C}$ for $3 \mathrm{~h}$ in a nitrogen environment; the results, not shown here for the sake of brevity, indicate that the effect of post-curing on $T_{g}$ is not significant, signifying that the dominant crosslinking reaction is associated with the amine/epoxy reaction. The same conclusion was drawn by Bell [25], for a DGEBA resin cured with an aromatic diamine, and by Kolar and Svitilova [26], neither of whom reported extensive reactions between the hydroxyl and epoxy groups.

The increase in the heat capacity $\left(\Delta C_{p}\right)$ across the glass transition is shown in Figure 6 as a function of $H P$. For amine rich samples, $\Delta C_{p}$ increases monotonically with increasing HP. This trend might be attributed to the flexibility of the hardener molecules compared with the resin molecules; the latter contain bulky epoxy and phenol groups. At high $H P$, some of the amine groups react with only one of their hydrogen atoms, see Figure 5c, which produces relatively flexible linear crosslink nodes. This increases rotational and conformational motions brought by the rubbery state in amine rich

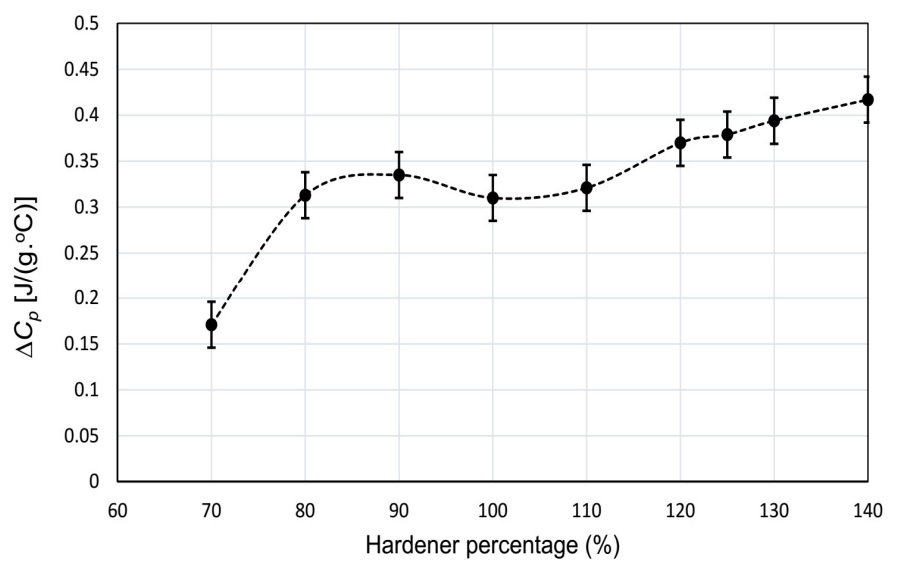

Figure 6. The change in the heat capacity over the glass transition process as a function of $H P$.

formulations. For the same ER system, other workers [5, 27] observed an increase in $\Delta C_{p}$ when increasing the length of the hardener molecules, i.e. increasing the number of the oxypropylene units in Figure 1b, which is in line with the trend obtained here for amine rich formulations. For resin rich samples, $\Delta C_{p}$ appears to increase slightly at $H P=90 \%$ before decreasing at $H P=80 \%$, albeit that these variations are within the uncertainties shown. Thereafter, $\Delta C_{p}$ decreases sharply at $H P=70 \%$. The data are not shown for $H P=60 \%$, since the DSC used cannot give reliable information near room temperature. The initially slight increase in $\Delta C_{p}$ for $H P=90 \%$ might be a reflection of a marked reduction in the crosslinking density at this formulation. However, on further reducing $H P$, the network will mainly consist of relatively rigid resin molecules, which allows less segmental motion upon entering the rubbery state. A comparable relation between $\Delta C_{p}$ and hardener ratio was reported by Calventus et al [3] for DEGBA epoxy cured with a similar polyether amine hardener.

The glass transition width, $\Delta T_{g}$, for all formulations is displayed in Figure 7. The transition width increases for the formulations with hardener percentage far away from $100 \%$ on both sides. This reflects increasing heterogeneity in networks with either excess epoxy or excess amine. For excess amine formulations, this heterogeneity can be detected from the illustrations in Figure 5c and 5d, where the distance between neighboring network nodes may be as short as one molecule or as long as a sequence of resin and hardener molecules. A

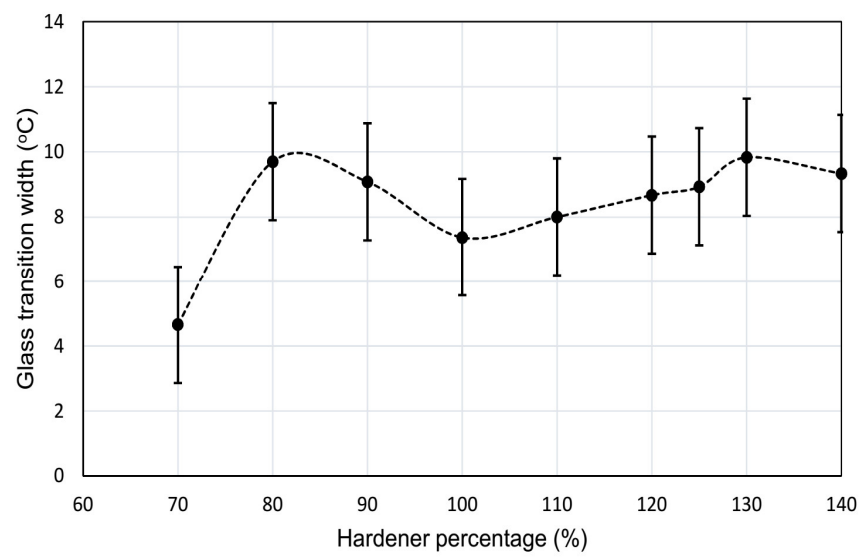

Figure 7. The width of the glass transition as a function of $H P$. 
comparable explanation can be proposed when $80 \%<H P<100 \%$, where competition for the available amine moieties leads to local variations in crosslinking density. A similar general form of behavior to that described above was reported by Gupta et al [28]. In this case, the glass transition process was examined using dynamic mechanical analysis and found to broadening, notably for hardener rich samples. However, for $H P=70 \%$, Figure 7 reveals a sharp decrease in $\Delta T_{g}$, which implies the formation of a more homogenous molecular structure or, more likely, that as the crosslink density drops (see Figure 5a), so the system is able to act in a more cooperative manner. That is, the observed glass transition is influenced by both structural and dynamical factors.

The enthalpy relaxation area for all samples is shown in Figure 8 as a function of $H P$. Comparing these data with the $T_{g}$ results shown in Figure 3 reveals that the variation in enthalpy relaxation is the inverse of the variation in $T_{g}$ and, correspondingly, in the crosslinking density. Similar trends have also been reported by other workers $[3,19]$. In the rubbery state (i.e. prior to vitrification during curing), the polymer segments have sufficient energy to move away from their equilibrium positions and, consequently, the lower the crosslinking density, the further the segments can be displaced. Consequently, at low crosslinking densities, the polymer segments need more energy to return to their equilibrium positions, which explains the trend observed in Figure 8.

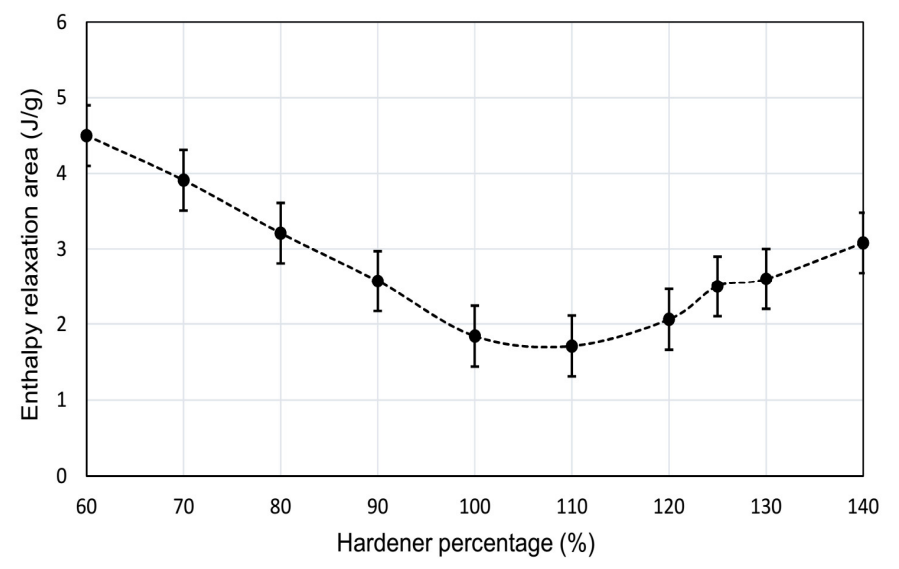

Figure 8. The enthalpy relaxation area as a function of $H P$.

\subsection{DIELECTRIC SPECTRA}

The effect of changing the stoichiometry on the dielectric spectrum is shown in Figure 9 for epoxy rich samples at room temperature. For the sample corresponding to the theoretically ideal stoichiometric formulation, $H P=100 \%$, a broad $\beta$ relaxation process can be observed starting at frequencies higher than $1 \mathrm{MHz}$ and extending down to frequencies around $10 \mathrm{~Hz}$. The breadth of this relaxation suggests that it is related to a distribution of segmental motions with different activation energies, where the motions with low activation energy are responsible for the high-frequency part of the relaxation and the motions with high activation energy are responsible for the lowfrequency part [29]. The peak of this relaxation is at approximately $4 \times 10^{4} \mathrm{~Hz}$ and its strength is $\sim 0.6$ (evaluated by calculating the increase in $\epsilon_{r}^{\prime}$ between $1 \mathrm{MHz}$ and $10 \mathrm{~Hz}$ ). The $\beta$ relaxation also appears in the epoxy rich samples, albeit that both its strength and breadth monotonically decrease with reducing amount of hardener, as shown in Figure $9 \mathrm{~b}$. Additionally, the peak of this relaxation moves slightly to higher frequencies. Comparable trends for the relaxation strength and breadth have been reported for similar epoxy/amine systems when reducing the crosslinking density $[6,30]$. Many studies have claimed that the $\beta$ relaxation in such systems is associated with the crankshaft rotation of the hydroxyether group $\left(-\mathrm{CH}(\mathrm{OH})-\mathrm{CH}_{2}-\mathrm{O}-\right)$ that is generated at each crosslink point due to the reaction between the epoxy and amine groups $[6,30,31]$. Based on this assumption, the $\beta$ relaxation strength should have a direct relation with the crosslinking density. Thus, at $H P=100 \%$, the $\beta$ relaxation should have the highest strength, which agrees with the results presented in Figure 9b. From the analysis conducted to produce Figure 5, the number of crosslinking junctions (epoxy/amine reactions) per unit volume of the cured sample was estimated as a function of $H P$ and presented in Figure 10 after normalizing it to its maximum at $H P=100 \%$. Also illustrated in Figure 10, the strength of the $\beta$ relaxation, which is derived from the measured spectrum for each formulation. The strength of the $\beta$ relaxation correlates with the density of epoxy/amine reaction for epoxy rich samples. However, the reduction in the $\beta$ relaxation strength is sharper than that predicted by the concentration of the crosslinking nodes. This might be caused by the reduction of the activation energy of some of the segmental motions that contribute to this relaxation with reducing the crosslinking density [29], which suggests that part of the relaxation moves to higher frequencies. Two consequences of this explanation would be expected. First, the strength of the low-frequency component of the relaxation should decrease with decreasing crosslinking density, which is

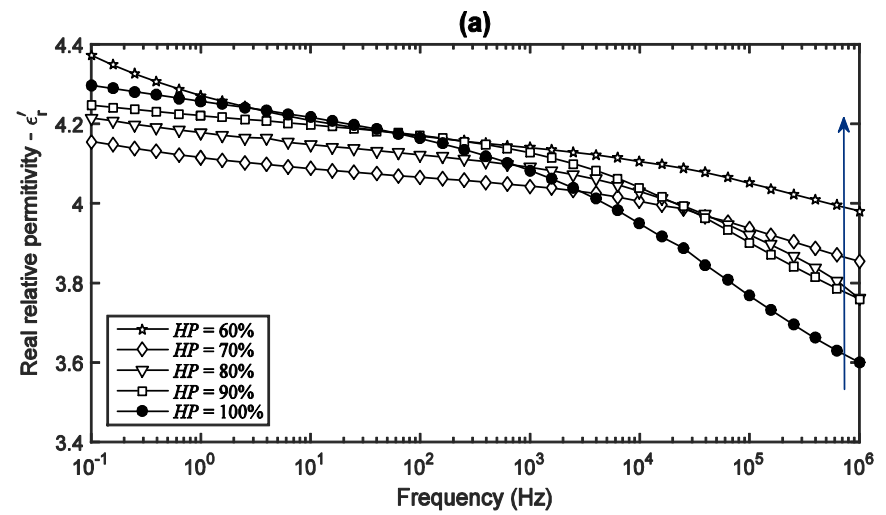

(b)

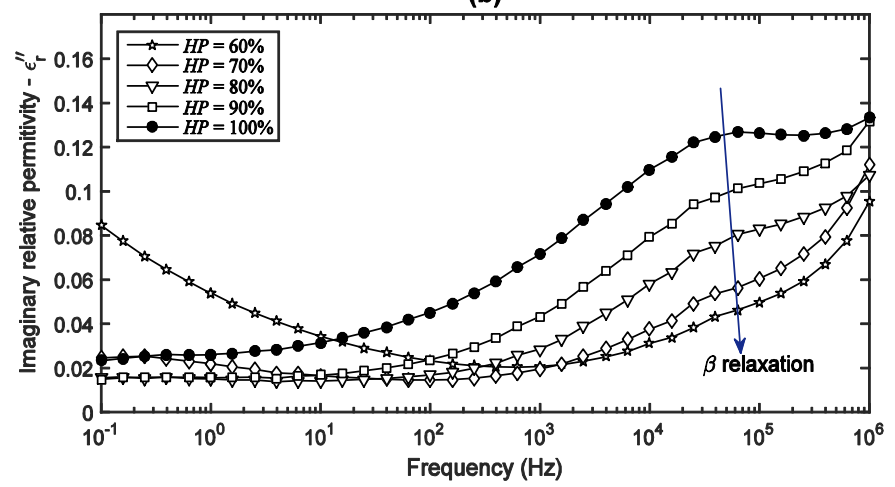

Figure 9. The dielectric spectra for epoxy rich samples at room temperature. 


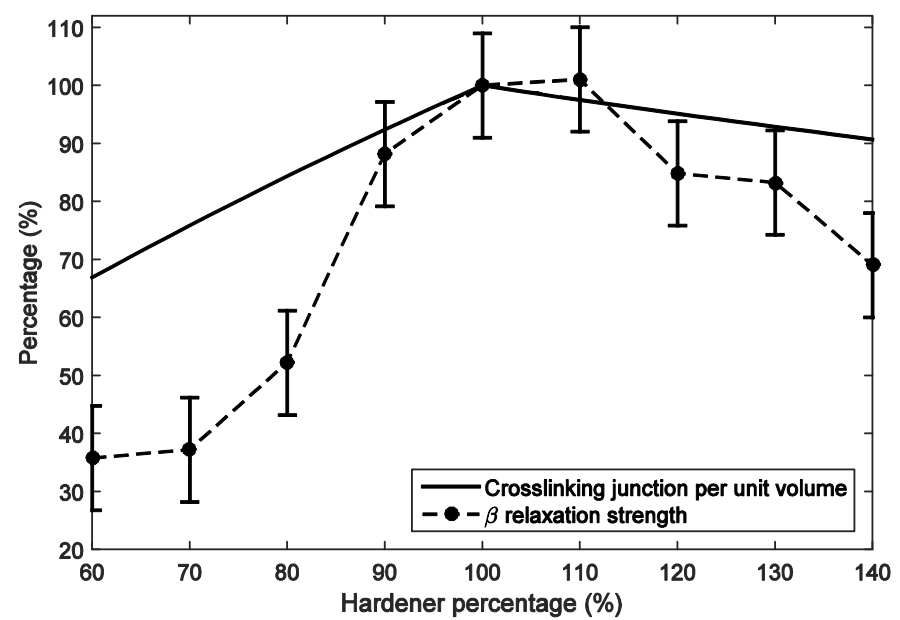

Figure 10. The number of the crosslinking junctions per unit volume and the $\beta$ relaxation strength normalized to their maximum at $H P=100 \%$.

the case in Figure 9. Second, part of the relaxation should move to higher frequencies and might manifest itself as a new relaxation process or as an extension of the existing $\beta$ relaxation to higher frequencies, i.e. beyond the range accessible here. Indeed mechanical spectroscopy results described in [5] indicate the emergence of a new relaxation peak beside the $\beta$ relaxation at a lower temperature for loosely crosslinked systems. This explanation is also in line with the gradual increase in the real relative permittivity at $1 \mathrm{MHz}$ from around 3.6 for $H P=100 \%$ to around 4 for $H P=60 \%$.

Another relaxation process starts appearing for $H P=70 \%$ and is clearly visible for $H P=60 \%$ in Figure $9 \mathrm{~b}$. The $T_{g}$ for these two samples is close to the spectroscopy measurement temperature $\left(\sim 23^{\circ} \mathrm{C}\right)$, which indicates that this relaxation process is associated with the main relaxation process occurring at the glass transition, the $\alpha$ relaxation.

Concerning the real part of the permittivity $\left(\epsilon_{r}^{\prime}\right)$, at low frequencies, it decreases as $H P$ falls from $100 \%$ to $70 \%$, as shown in Figure 9a. This reduction can be ascribed to the reduction in the density of hydroxyether groups, as discussed earlier. Hence, decreasing $H P$ from $100 \%$ to $70 \%$ results in a reduction in the $\beta$ relaxation strength and, correspondingly, the magnitude of $\epsilon_{r}^{\prime}$ of the resulting network. However, at $H P=60 \%, \epsilon_{r}^{\prime}$ increases to around the same values as for the sample where $H P=100 \%$. This might be a result of the enhancement of the contributions from other moieties enabled by the dramatic reduction in the crosslinking, particularly since, at the measurement temperature, the network is not far from its rubbery state for $H P=60 \%$. This could be deduced from the appearance of the $\alpha$ relaxation for $H P=60 \%$.

Dielectric spectra for amine rich samples are shown in Figure 11. As can be predicted from the trends discussed above in connection with Figure 10, the $\beta$ relaxation strength smoothly decreases as HP increases from $100 \%$ to $140 \%$, due to the consequent reduction in the hydroxyether group density. Similar to what was stated for epoxy rich samples, the decrease in the $\beta$ relaxation strength is sharper than would be expected by estimating the hydroxyether group density. This again refers to the movement of part of the $\beta$ relaxation to higher frequencies for loosely crosslinked networks. No $\alpha$ relaxation process is observed for the amine rich samples, since none of these samples is close to its $T_{g}$ during these measurements.

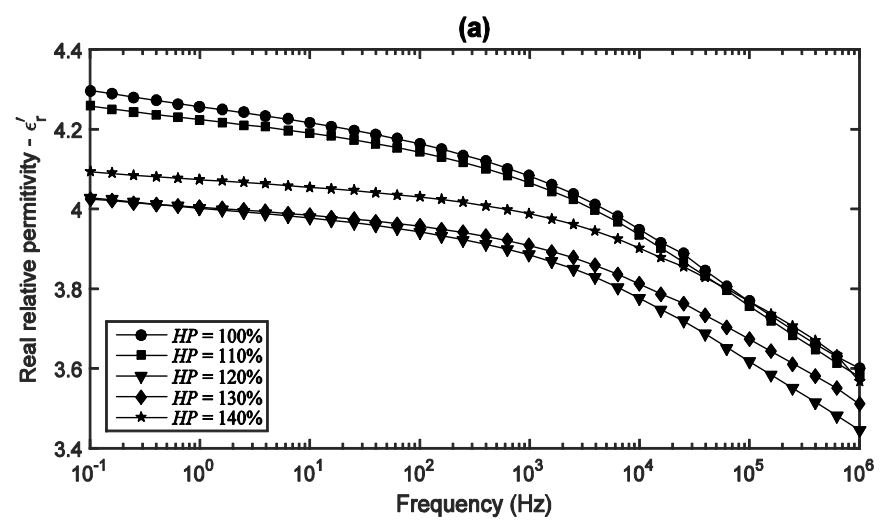

(b)

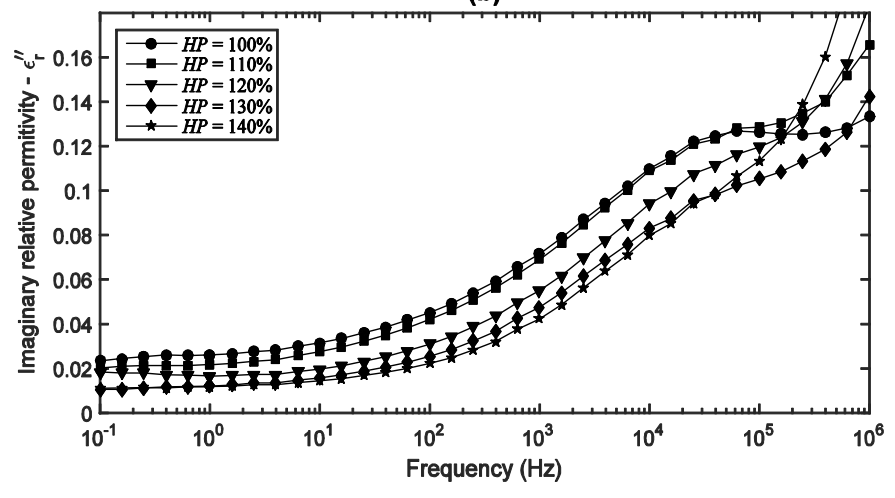

Figure 11. The dielectric spectra for amine rich samples at room temperature

\subsection{CONDUCTIVITY}

The effect of stoichiometry on charge transport was determined by measuring the DC conductivity at an applied field of $42 \mathrm{kV} / \mathrm{mm}$ at temperatures up to $60^{\circ} \mathrm{C}$ (Figure 12). At room temperature, when all samples are well in their glassy state, these data indicate that the DC conductivity increases steadily with increasing $H P$. Such behavior may be associated with either or both of two factors that are affected by changing $H P$ : the network structure; the chemical constituents of the network. As established earlier, increasing or decreasing $H P$ beyond the stoichiometric ratio (i.e. $H P=100 \%$ ) results in a less crosslinked network with enhanced segmental dynamics at a given temperature, which means that the network structure does not monotonically change with $H P$. Since the measured DC conductivity increased monotonically with increasing $H P$ from $60 \%$ to $140 \%$, it cannot be correlated with the variation that occurs in the network dynamics or architecture. Therefore, we suggest that, in the glassy state, the DC conductivity of the epoxy networks should be primarily related to their chemical content. Virtanen et al [32] studied the impact of introducing electroactive groups (i.e. modifying the chemical content) into an epoxy matrix by adding two types of surface modified silica nanoparticles. Particles functionalized with long epoxy compatible molecules resulted in improved particle dispersion, but had only a marginal effect on electrical behavior. Treating the same particles with short electroactive molecules, namely oligothiophene and ferrocene, produced a substantial beneficial influence on dielectric performance. The authors argued that the electroactive molecules offer new energy states that could trap 
electrons and, thus, affect charge transport dynamics within the system. A similar conclusion was drawn by Siddabattuni et al [33], who found that the inclusion of nanoparticles functionalized with electron-withdrawing functional groups led to a nanocomposite with reduced DC conductivity, whereas the inclusion of electron-donating functional groups, such as amine groups, resulted in increased DC conductivity. These studies suggest that the electrical behavior of a material is sensitive to the electroactivity of the system. In the systems investigated here, the main electroactive groups, which are affected by modifying $H P$, are the hydroxyl and the amine groups. The concentration of hydroxyl groups was discussed and shown in Figure 10 as a function of $H P$. The amine groups mainly exist in the amine rich formulations and their concentration increases with increasing HP. Consequently, the slight decrease in the DC conductivity for epoxy rich samples may be associated with the lower concentration of hydroxyl group. For amine rich samples, since the hydroxyl content decreases as $H P$ increases, the significant rise in the DC conductivity might be related to the increase in the amine group concentration in these samples.

Considering the DC conductivity and dielectric spectroscopy results in concert suggests that while the polar amine groups do not obviously affect the dielectric constant of the material, they significantly influence charge transport. Being part of the backbone chains that make up the network, the amine groups will possess limited rotational/conformational mobility and, consequently, it will be difficult for them to reorient in response to the electric field. This, therefore, limits their contribution to the orientation polarization and, hence, to the permittivity. Conversely, the electroactive character of the amine and hydroxyl groups might alter the local density of states [34] in a way that increases the electronic conductivity without the need for any rotational or conformational motions. This again emphasizes that the dielectric performance is more sensitive to the electroactive character of the network constituents rather than to its segmental dynamics.

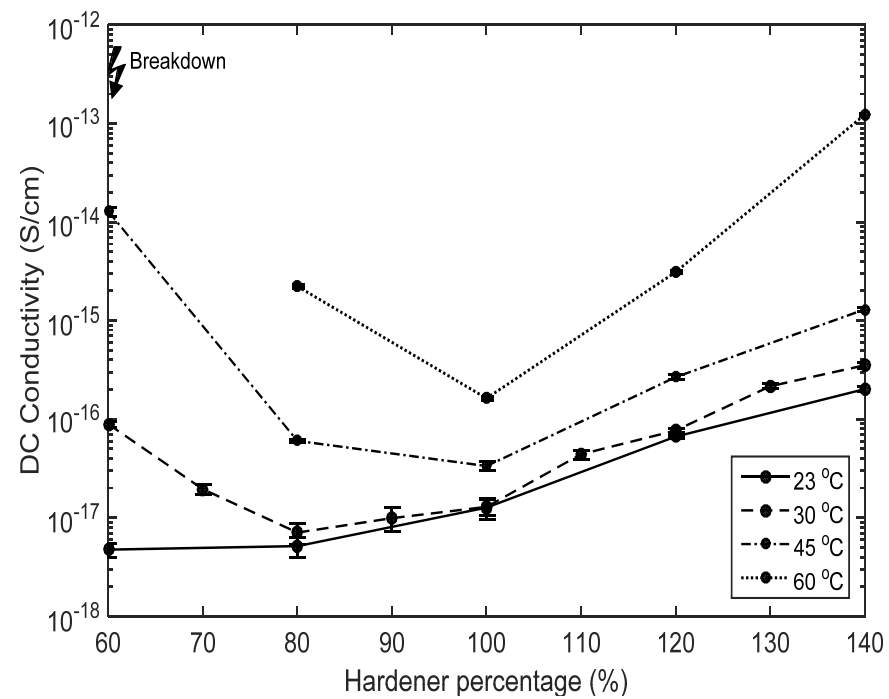

Figure 12. DC conductivity averaged over two hours at different temperatures and applied field of $42 \mathrm{kV} / \mathrm{mm}$, breakdown occurred at $60{ }^{\circ} \mathrm{C}$ for $H P=60 \%$.
Figure 12 shows that increasing the temperature results in an increase in the DC conductivity, however, the relation is not linear. As the temperature approaches $T_{g}$ for the particular sample, the DC conductivity increases much more sharply. Being close to the rubbery state enhances the movement of any ionic residues available within the network, such as $\mathrm{Na}^{+}$and $\mathrm{Cl}^{-}$ ions which are generated as by-products during the synthesis process of many commercial DGEBA resins [35]. Hence, as the material goes into its rubbery state, the contribution from ionic conductivity increases and, consequently, the whole DC conductivity increases.

\subsection{AND AC BREAKDOWN STRENGTH}

DC breakdown data obtained from selected samples are plotted assuming a two-parameter Weibull distribution in Figure 13. The Weibull scale and shape parameters for these samples at the 63.2 percentile with error bars that represent the $95 \%$ confidence bounds are shown in Figure 14. For $H P>100 \%$, the DC breakdown strength decreases monotonically with increasing amine content while, for samples with $H P<100 \%$, the Weibull scale parameter

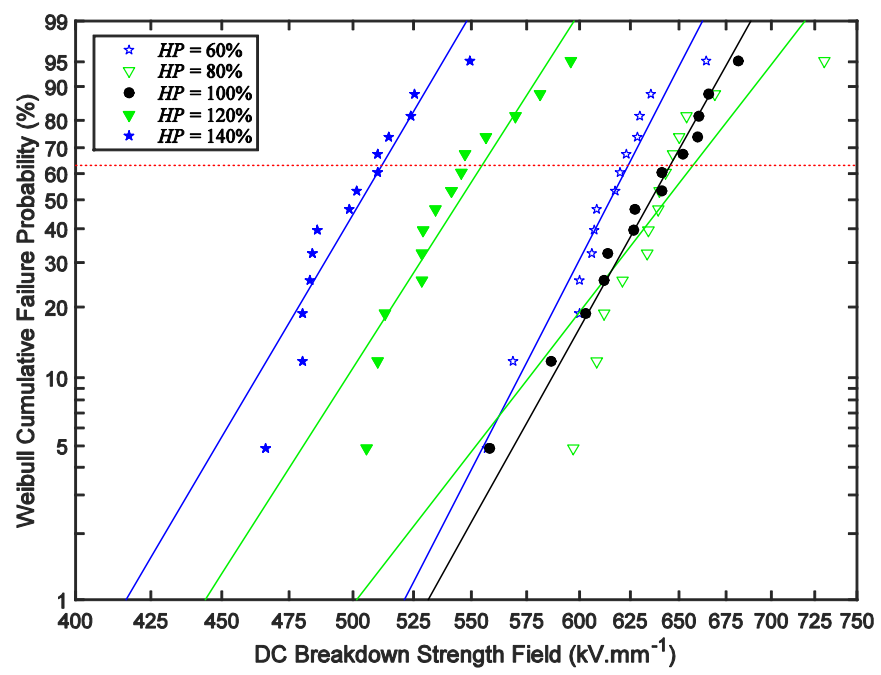

Figure 13. DC breakdown strength for selected samples.
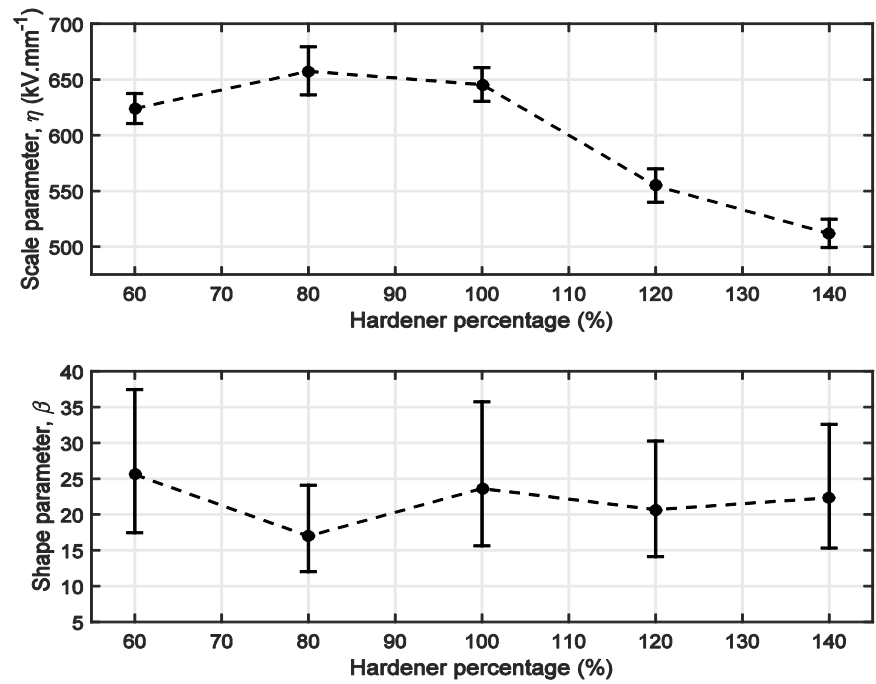

Figure 14. DC Weibull scale parameter (top) and shape parameter (bottom) as a function of $H P$, the error bars indicate the $95 \%$ confidence bounds. 
increases slightly with decreasing $H P$ up to $80 \%$ before decreasing for $H P=60 \%$, albeit that these latter variations are not statistically significant. These results broadly correlate with the DC conductivity measured at room temperature, as breakdown testing was also performed at room temperature. Being well below $T_{g}$, the DC conductivity and, consequently, the DC breakdown strength are mainly affected by the hydroxyl and amine content, this is applicable to samples with $H P \geq 80 \%$. On the other hand, by approaching the rubbery state, the DC conductivity might be enhanced by other mechanisms such as enabling more ionic conduction as could be revealed from the results at $H P=60 \%$.

The effect of changing the hardener percentage on the $\mathrm{AC}$ breakdown strength is shown in Figure 15 and the Weibull parameters for all samples are presented Figure 16 with error bars that indicate the $95 \%$ confidence bounds. There are no significant changes to the Weibull scale parameters of the measured samples, which range between 210 and $226 \mathrm{kV} / \mathrm{mm}$ with overlapping confidence bounds. Thus, these results do not

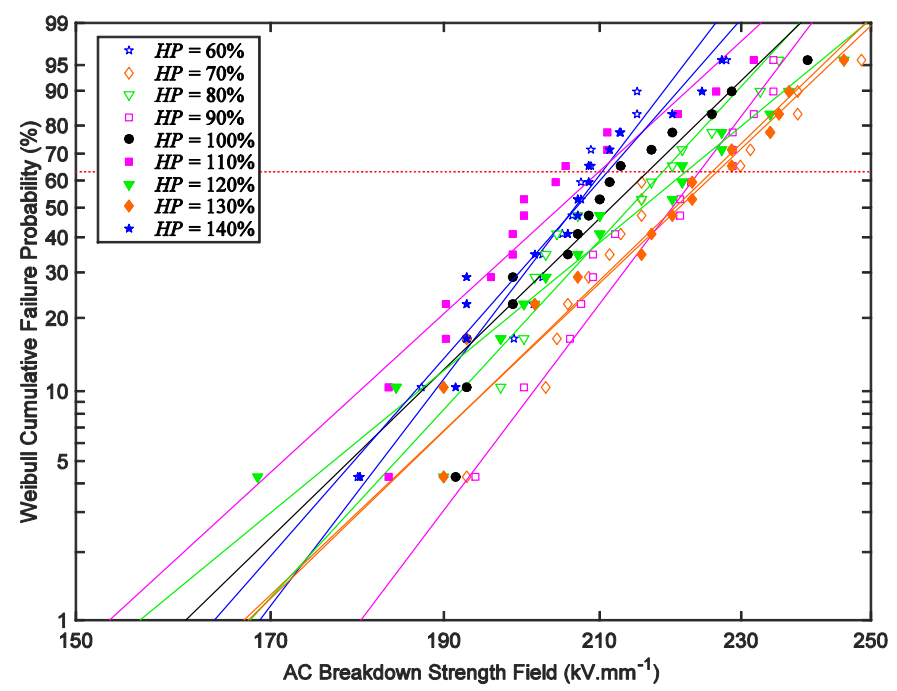

Figure 15. AC breakdown strength for all samples.
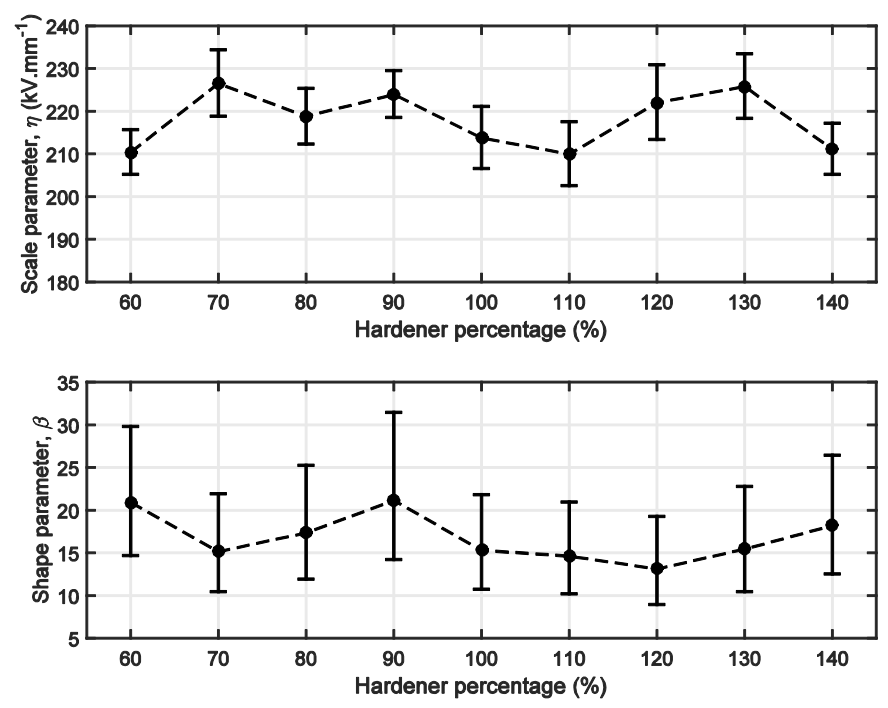

Figure 16. Weibull scale parameter (top) and shape parameter (bottom) versus hardener percentage, the error pars are based on $95 \%$ confidence bounds. support any correlation between the AC breakdown strength and the material formulation within the composition range considered here. Unlike the DC breakdown strength, which broadly correlates with the DC conductivity, the AC breakdown strength seems to be unaffected by variations in the DC conductivity, which implies that the $\mathrm{AC}$ and $\mathrm{DC}$ breakdown strengths are controlled by different mechanisms. This conclusion is in line with other experimental findings [36-39], which also show that the AC breakdown strength does not correlate with variations in DC conductivity or DC breakdown strength. Grzybowski [36] studied the impact of water absorption on AC and DC breakdown strengths of polyethylene terephthalate and found that, compared with the sharp reduction in the DC breakdown strength, the AC breakdown strength only showed a relatively slight reduction due to water absorption. This implies that the uniform distribution of water molecules inside the insulation material has a more detrimental effect on DC breakdown than on AC breakdown. Huang [37] reported that adding surface treated nanoparticles into a polyethylene matrix reduced the $\mathrm{AC}$ breakdown strength, even though it decreased the DC conductivity to be lower than that of the unfilled matrix. The authors attributed this behavior to the defects and free volume that were introduced by the addition of the nanoparticles. This is in accordance with the finding in [38], which indicated that the $\mathrm{AC}$ breakdown strength is more sensitive to the presence of defects or deficiencies in insulation materials. In the systems investigated here, since the amine and hydroxyl groups, which affect the conductivity, are uniformly distributed throughout the material, they should not produce defects and, thus, should not severely affect the AC breakdown strength.

\section{CONCLUSIONS}

Changing the stoichiometry showed that as long as the epoxy network is not near the glass transition temperature, its electrical behavior is mainly related to the chemical content, represented by the amine and hydroxyl groups, rather than to the network structure of the cured epoxy. Increasing the hardener (amine) content beyond the optimal stoichiometry has a detrimental effect on electrical properties like DC conductivity and DC breakdown strength. On the other hand, decreasing the amine content has no adverse consequences for the examined electrical properties, particularly when the material is well within its glassy state. However, consequent changes to the network structure will affect $T_{g}$ and, as the temperature approaches the $T_{g}$ of the material, increased ionic conduction will play an increasingly important role.

Unlike the DC breakdown strength which correlates with the DC conductivity, the AC breakdown strength is not significantly influenced by the change in the DC conductivity, which implies that a homogenous increase in the DC charge transport does not necessarily lead to a noticeable change in the short-term AC breakdown strength.

\section{ACKNOWLEDGEMENTS}

The authors would like to thank Dr Matt Praeger for his help with dielectric spectroscopy measurements and also Dr Suvi 
Virtanen for her help with FTIR measurements.

\section{REFERENCES}

[1] "Market Report: Global Epoxy Resin Market," Acmite Market Intelligence, 2014.

[2] I. Hamerton, Recent Developments in Epoxy Resins: Rapra Technology Ltd, 1997.

[3] Y. Calventus, S. Montserrat, and J. M. Hutchinson, "Enthalpy relaxation of non-stoichiometric epoxy-amine resins," Polymer, vol. 42, pp. 70817093, Jul 2001.

[4] R. J. Morgan, F.-M. Kong, and C. M. Walkup, "Structure-property relations of polyethertriamine-cured bisphenol-a-diglycidyl ether epoxies," Polymer, vol. 25, pp. 375-386, 1984

[5] P. N. Patil, S. K. Rath, S. K. Sharma, K. Sudarshan, P. Maheshwari, M. Patri, S. Praveen, P. Khandelwal, and P. K. Pujari, "Free volumes and structural relaxations in diglycidyl ether of bisphenol-A based epoxypolyether amine networks," Soft Matter, vol. 9, pp. 3589-3599, 2013.

[6] C. Jordan, J. Galy, and J. P. Pascault, "Measurement of the extent of reaction of an epoxy-cycloaliphatic amine system and influence of the extent of reaction on its dynamic and static mechanical-properties," Journal of Applied Polymer Science, vol. 46, pp. 859-871, Oct 151992.

[7] V. B. Gupta and C. Brahatheeswaran, "Molecular packing and freevolume in cross-linked epoxy networks," Polymer, vol. 32, pp. 18751884,1991

[8] F. Meyer, G. Sanz, A. Eceiza, I. Mondragon, and J. Mijovic, "The effect of stoichiometry and thermal history during cure on structure and properties of epoxy networks," Polymer, vol. 36, pp. 1407-1414, Mar 1995

[9] G. R. Palmese and R. L. McCullough, "Effect of epoxy amine stoichiometry on cured resin material properties," Journal of Applied Polymer Science, vol. 46, pp. 1863-1873, 1992.

[10] V. T. Nguyen, A. S. Vaughan, P. L. Lewin, and A. Krivda, "The Effect of Resin Stoichiometry and Nanoparticle Addition on Epoxy/Silica Nanodielectrics," IEEE Transactions on Dielectrics and Electrical Insulation, vol. 22, pp. 895-905, 2015.

[11] C. Yeung and A. S. Vaughan, "On the effect of nanoparticle surface chemistry on the electrical characteristics of epoxy-based nanocomposites," Polymers, vol. 8, 2016.

[12] M. Reading, A. S. Vaughan, and P. L. Lewin, "An investigation into improving the breakdown strength and thermal conduction of an epoxy system using boron nitride," 2011 IEEE Conference on Electrical Insulation and Dielectric Phenomena, pp. 636-9, 2011.

[13] W. Zhou, J. Zuo, X. Zhang, and A. Zhou, "Thermal, electrical, and mechanical properties of hexagonal boron nitride-reinforced epoxy composites," Journal of Composite Materials, vol. 48, pp. 2517-2526, 2014.

[14] F. N. Alhabill, T. Andritsch, and A. S. Vaughan, "Effect of the processing method on the electrical behavior of silicon nitride/epoxy nanocomposites," 2015 IEEE Conference on Electrical Insulation and Dielectric Phenomena, pp. 511-514, 2015.

[15] T. Andritsch, R. Kochetov, Y. T. Gebrekiros, P. H. F. Morshuis, and J. J. Smit, "Short term DC breakdown strength in epoxy based BN nano- and microcomposites," 10th IEEE International Conference on Solid Dielectrics, pp. 1-4, 2010.

[16] D. Qiang, M. He, G. Chen, and T. Andritsch, "Influence of nano-SiO2 and $\mathrm{BN}$ on space charge and $\mathrm{AC} / \mathrm{DC}$ performance of epoxy nanocomposites," IEEE Electrical Insulation Conference (EIC), pp. 492-495, 2015.

[17] S. G. Prolongo, A. Salazar, A. Urena, and J. Rodriguez, "Effect of hydroxyl content on the morphology and properties of epoxy/poly(styrene-co-allylalcohol) blends," Polymer Engineering and Science, vol. 47, pp. 1580-1588, Oct 2007.

[18] M. F. Grenierloustalot and P. Grenier, "The role of impurities in the mechanisms and kinetics of epoxy-resins and their effects on final resin properties," British Polymer Journal, vol. 22, pp. 303-313, 1990.

[19] J. M. Morancho and J. M. Salla, "Relaxation in partially cured samples of an epoxy resin and of the same resin modified with a carboxyl-terminated rubber," Polymer, vol. 40, pp. 2821-2828, 1999.

[20] C. L. Soles, F. T. Chang, B. A. Bolan, H. A. Hristov, D. W. Gidley, and A. F. Yee, "Contributions of the nanovoid structure to the moisture absorption properties of epoxy resins," Journal of Polymer Science Part B-Polymer Physics, vol. 36, pp. 3035-3048, Dec 1998.
[21] G. Nikolic, S. Zlatkovic, M. Cakic, S. Cakic, C. Lacnjevac, and Z. Rajic, "Fast Fourier Transform IR Characterization of Epoxy GY Systems Crosslinked with Aliphatic and Cycloaliphatic EH Polyamine Adducts," Sensors, vol. 10, pp. 684-696, Jan 2010.

[22] D. L. Pavia, G. M. Lampman, G. S. Kriz, and J. R. Vyvyan, Introduction to Spectroscopy, 5 ed.: Cengage Learning, 2013.

[23] C. Sisbandini, D. Brandell, T. Gustafsson, and L. Nyholm, "The Mechanism of Capacity Enhancement in LiFePO4 Cathodes Through Polyetheramine Coating," Journal of the Electrochemical Society, vol. 156, pp. A720-A725, 2009.

[24] T. Y. Lo, Y. J. Wang, D. M. Liu, and W. T. Whang, "Surface characteristics and biofunctionality of a novel high-performance, hydrophilic Jeffamine-added fluoro-containing polyimide for biomedical applications," Journal of Polymer Research, vol. 22, 2015.

[25] J. P. Bell, "Structure of a typical amine-cured epoxy resin," Journal of Polymer Science Part A-2-Polymer Physics, vol. 8, pp. 417-436, 1970

[26] F. Kolar and J. Svitilova, "Kinetics and mechanism of curing epoxy/anhydride systems," Acta Geodynamica Et Geomaterialia, vol. 4, pp. 85-92, 2007.

[27] S. Montserrat, P. Cortes, Y. Calventus, and J. M. Hutchinson, "Effect of crosslink length on the enthalpy relaxation of fully cured epoxy-diamine resins," Journal of Polymer Science Part B-Polymer Physics, vol. 38, pp. 456-468, 2000.

[28] A. P. Gupta, S. Ahmad, and A. Dev, "Development of Novel Bio-Based Soybean Oil Epoxy Resins as a Function of Hardener Stoichiometry," Polymer-Plastics Technology and Engineering, vol. 49, pp. 657-661, 2010

[29] L. Heux, J. L. Halary, F. Laupretre, and L. Monnerie, "Dynamic mechanical and C-13 nmr investigations of molecular motions involved in the beta relaxation of epoxy networks based on DGEBA and aliphatic amines," Polymer, vol. 38, pp. 1767-1778, Apr 1997.

[30] C. L. Soles and A. F. Yee, "A discussion of the molecular mechanisms of moisture transport in epoxy resins," Journal of Polymer Science Part BPolymer Physics, vol. 38, pp. 792-802, Mar 12000.

[31] T. V. Kosmidou, A. S. Vatalis, C. G. Delides, E. Logakis, P. Pissis, and G. C. Papanicolaou, "Structural, mechanical and electrical characterization of epoxy-amine/carbon black nanocomposites," Express Polymer Letters, vol. 2, pp. 364-372, May 2008.

[32] S. Virtanen, T. M. Krentz, J. K. Nelson, L. S. Schadler, M. Bell, B. Benicewicz, H. Hillborg, and S. Zhao, "Dielectric breakdown strength of epoxy bimodal-polymer-brush-grafted core functionalized silica nanocomposites," IEEE Transactions on Dielectrics and Electrical Insulation, vol. 21, pp. 563-570, Apr 2014.

[33] S. Siddabattuni, T. P. Schuman, and F. Dogan, "Dielectric Properties of Polymer-Particle Nanocomposites Influenced by Electronic Nature of Filler Surfaces," ACS Applied Materials \& Interfaces, vol. 5, pp. 19171927, 2013

[34] M. Meunier, N. Quirke, and A. Aslanides, "Molecular modeling of electron traps in polymer insulators: Chemical defects and impurities," Journal of Chemical Physics, vol. 115, pp. 2876-2881, 2001.

[35] H. Kim and K. Char, "Dielectric changes during the curing of epoxy resin based on the diglycidyl ether of bisphenol A (DGEBA) with diamine," Bulletin of the Korean Chemical Society, vol. 20, pp. 1329-1334, Nov 1999.

[36] S. Grzybowski, E. A. Feilat, P. Knight, and L. Doriott, "Breakdown voltage behavior of PET thermoplastics at DC and AC voltages," IEEE Southeastcon '99, Proceedings, pp. 284-287, 1999.

[37] X. Y. Huang, F. Liu, and P. K. Jiang, "Effect of Nanoparticle Surface Treatment on Morphology, Electrical and Water Treeing Behavior of LLDPE Composites," IEEE Transactions on Dielectrics and Electrical Insulation, vol. 17, pp. 1697-1704, 2010

[38] B. K. Gupta, "Use of AC and DC hipot tests to assess condition of stator insulation," Proceedings: Electrical Electronics Insulation Conference and Electrical Manufacturing \& Coil Winding Conference, IEEE, pp. 605608, 1995.

[39] G. Iyer, R. S. Gorur, R. Richert, A. Krivda, and L. E. Schmidt, "Dielectric Properties of Epoxy based Nanocomposites for High Voltage Insulation," IEEE Transactions on Dielectrics and Electrical Insulation, vol. 18, pp. 659-666, 2011. 


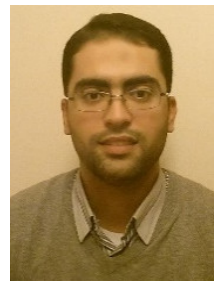

Fuad N. Alhabill received his BSc (with distinction) in 2008 from the Islamic University of Gaza, Palestine, where he worked as a teaching assistant and lecturer until 2011. He also worked as an electrical engineer at the Ministry of Telecommunication, Palestine, for 3 years before he was awarded Said Foundation scholarship and received MSc (with distinction) in Energy \& Sustainability with E.P.E. from University of Southampton, UK, in 2014. Currently he is pursuing his $\mathrm{PhD}$ study in high voltage power engineering at University of Southampton, UK.

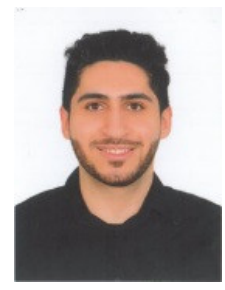

Raed Ayoob was born in Bahrain in 1992, and moved to the UK in 2010. He received the B.Eng degree in Electrical engineering in 2013 and is currently pursuing his Ph.D degree in High Voltage Electrical Engineering at the University of Southampton. His Ph.D research project focuses on the use of polymer nanocomposites as insulation materials in high voltage cables.

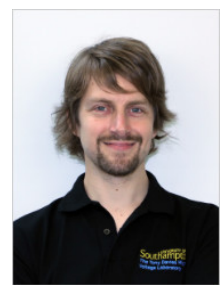

Thomas Andritsch (M'11) was born in Innsbruck, Austria in 1980. He received the Dipl.-Ing. Degree in Electrical Engineering from Graz University of Technology in 2006 and his $\mathrm{PhD}$ in the same field from Delft University of Technology in 2010 . He is currently working as Lecturer at the University of Southampton with focus on advanced and materials for high voltage applications \& plant. He has extensive experience with preparation and testing of polymer-based electrical insulation materials, including nanodielectrics, electroactive polymers and syntactic foams, as well as liquid insulation systems and nanofluids. Thomas is currently the chair of the UK \& Ireland IEEE DEIS Chapter. He is member of the IEEE DEIS working group on nanodielectrics, developing recommended practices for nanocomposite synthesis and preparation.

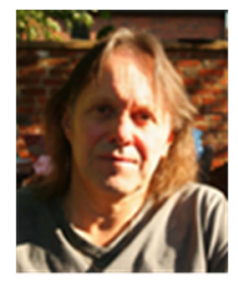

Alun Vaughan (SM'07) has a B.Sc. degree in chemical physics and a Ph.D. degree in polymer physics. After working at the UK's Central Electricity Research Laboratories and spending a period as an academic at The University of Reading, he is now Professor of Dielectric Materials at the University of Southampton. He is a former chair of The Dielectrics Group of the Institute of Physics, a Fellow of the Institute of Physics, a Fellow of the IET and delivered the 2016 IEEE DEIS Eric O.

Forster memorial lecture. 
